\title{
GW23-e2138 THE RELATIONSHIP BETWEEN ORAL HEALTH, DENTAL HABITS AND NEW ONSET OF HYPERTENSION, A 2 YEARS FOLLOW-UP STUDY
}

doi:10.1136/heartjnl-2012-302920e.7

Yang Fan, Wang Ruiying, Sun Yuting, Jing Yuejuan, Li Taotao, Li Yafeng. The Second Hospital Of Shanxi Medical University

Objectives As we all know, cardiovascular disease is a kind of lifestyle related disease, but there is little study about the relationship between cardiovascular disease and oral health. Therefore, the objective of present study was to investigate the dental habits condition in an adult Taiyuan population, Shanxi province. And to study whether there is a relationship between oral care habits and hypertension.

Methods 1371 subjects (477 male and 894 female, aged 2877 years) who were from Yingze community participated physical examination and answered a questionnaire including medical history, lifestyle, dietary habit, oral health in 2008. All the subjects were followed up in 2010 and data including blood pressure (BP), fasting blood glucose (FPG), cholesterol (CHOL), triglyceride (TG), HDL-Cholesterol (HDL-C), LDL-Cholesterol (LDL-C), waist/hip ratio (WHR) and body mass index (BMI). We payed more attention to investigate new cases were diagnosed with hypertension in under 2 years. The subjects were categorised according to the oral health habits and we used $\chi^{2}$ test to analyse the relationship between oral health, dental habits and new onset of hypertension.

Results 1363 subjects (476 male and 887 female, aged 35-56 years) with complete examination data were included in this study. Compared with those who often use the toothpick, the subjects never use or use the toothpick sometimes had a lower proportion of developing hypertension $(23.2 \%$ vs $19.5 \%$ vs $35.3 \%, p=0.002)$. About $28.2 \%$ subjects never use mouthwash in this study and they

were tend to have a higher risk of developing hypertension $(25.8 \%$ vs $15.6 \%, p=0.019)$. And binary logistic regression analysis showed that the age of beginning to brush influenced the occurrence of hypertension after carefully adjusting for age, sex, body mass index, lifestyle factors (total physical activity, cigarette smoking, and alcohol consumption) ( $\mathrm{OR}=1.37495 \% \mathrm{CI} 1.148$ to 1.643 , $\mathrm{p}=0.001)$. The subjects brushed teeth at the age of $3-5$ tend to have a low rate of new onset hypertension.

Conclusions Brushing teeth regularly, such as keeping good oral care habits at the early age (3-5 years old), using mouthwash regularly and trying to avoid the use of toothpick, significantly reduces the risk of new onset hypertension in the Chinese population. 